\title{
Estrategia en la enseñanza de las ciencias para fortalecer la competencia "indaga" a través de la meteorología
}

\section{Strategy in the teaching of sciences to strengthen "inquiry" competence through meteorology}

\author{
July Ruth Rimac Coral ${ }^{1}$, David Esteban Espinoza ${ }^{2 a}$ \\ Institución Educativa San Cristóbal, Lima, Perú ${ }^{1}$ \\ Universidad Ricardo Palma, Lima, Perú ${ }^{2}$ \\ ORCID ID: https://orcid.org/0000-0003-0837-6312 1 \\ ORCID ID: https://orcid.org/0000-0001-8025-6409²
}

Recibido: 21 de febrero de 2021

Aceptado: 27 de junio de 2021

\begin{abstract}
Resumen
Los objetivos de la investigación son: i) describir la experiencia para la enseñanza de las ciencias, que permitió implementar el Servicio Meteorológico Escolar (SME) y ii) describir la estrategia de enseñanza de las ciencias que permite fortalecer la competencia de indagación a través del SME. El enfoque de la investigación fue cualitativo y su diseño la investigación acción. La experiencia contó con la participación de 22 estudiantes, 15 integrantes distribuidos entre el primer y quinto grado conformaron el SME y siete estudiantes de segundo grado participaron directamente de la estrategia indaga. Los hallazgos indican que los estudiantes transitan por todas las etapas de la estrategia indagación: problematización, diseña estrategias para indagar, genera y registra datos, analiza datos, evalúa y comunica. Finalmente se concluye que mediante la experiencia de innovación de la enseñanza de las ciencias fue posible implementar el SME. Además, la estrategia de la enseñanza de las ciencias permitió fortalecer la competencia indaga en los estudiantes participantes en el estudio.
\end{abstract}

Palabras clave: Estrategia, enseñanza, ciencias, competencia, indagación, meteorología. 


\begin{abstract}
The objectives of this investigation are i) describe the experience for science teaching, which enabled the implementing of the School Meteorological Service (SMS) and ii) describe the science teaching strategy that strengthens the research competence through the SME. The focus of the research was qualitative and its design the research in action. This experience was attended by twenty-two students, fifteen members distributed between the first and fifth grades were part of SME and seven students from second grade participated directly in the "inquire" strategy. Findings indicate that students travel through all stages of the inquiry strategy: raise a problem, designs strategies to research, generates and records data, analyzes data, evaluates and communicates them. Finally, it is concluded that through the experience of innovation in science teaching it was possible to implement the SME. In addition, the science teaching strategy made strengthen the competence "inquires" possible in the students participating in the study.
\end{abstract}

Keywords: Strategy, teaching, science, competition, inquiry, meteorology.

\title{
Introducción
}

El interés de las Academias de Ciencias del Mundo por una renovación en la enseñanza de las ciencias naturales, llevó a la creación de la metodología Enseñanza de las Ciencias Basada en la Indagación (ECBI), se reconoce su potencial al contribuir que los estudiantes desarrollen conceptos, competencias, actitudes e intereses en sociedades crecientemente dependientes de la aplicación de la ciencia (Harlen, 2013), además, de cambiar su percepción frente al aprendizaje de las ciencias (Molina-Ruiz y GonzalesGarcía, 2021)

El aprendizaje empleando la metodología ECBI se concreta a través de actividades que implican observar, formular preguntar, revisar fuentes de información y evidencias experimentales, planificar la indagación, proponer respuestas, explicaciones y comunicar resultados (Meisel et al., 2010). Por su lado, ECBI (2015) la delimita en cuatro etapas: focalización, exploración, reflexión y aplicación. Sin embargo, el objetivo común es desarrollar el conocimiento científico y la actitud científica.

En la búsqueda de mejorar la enseñanza de las ciencias se otorga importancia a desarrollar procesos de pensamiento autónomos en matemáticas a través de la implementación de secuencias didácticas basadas en ECBI (Sala \& Font, 2019), asimismo, se promueve la reflexión de los maestros, su disposición y actitudes hacia la enseñanza de las ciencias que incidan en el diseño de secuencias didácticas que promuevan la 
construcción propia de conocimientos en los estudiantes, en ese sentido se reporta que la ECBI modifica la percepción de los estudiantes sobre su aprendizaje de las ciencias, al preferir los aprendizajes vivenciales (Molina-Ruiz \& Gonzales-García, 2021). También se desarrollan experiencias con docentes, promoviendo su capacitación, seguimiento, evaluación y disponibilidad de materiales, aplicando el aprendizaje basado en la indagación (Crujeiras \& Cambeiros, 2018; Meisel et al., 2010; Pazuela \& Cascarroza, 2018).

También se reportan estudios en la búsqueda de analizar y mejorar el proceso de enseñanza-aprendizaje mediante la meteorología, con esta finalidad se propugna que el estudiante se familiarice con un lenguaje científico, demandando objetividad, orden, precisión y exactitud, que sean capaces de recoger datos del tiempo in situ, que interactúen con la naturaleza realizando observación directa de los fenómenos meteorológicos (Romagnoli \& Sebben, 2015). En estos trabajos se hace frente a la realidad desde lo cotidiano.

Se emplea el término indagación de dos maneras distintas. La primera se refiere a las habilidades de los estudiantes para formularse preguntas y su búsqueda por responderlas. La segunda a la estrategia de enseñanza - aprendizaje de conceptos científicos conducida por el docente, aprendiendo ciencia desde la ciencia y no de manera mecánica (National Academy of Sciences, 2000). En la perspectiva de Reyes-Cárdenas y Padilla (2012), la indagación es una estrategia que provee metodologías y estructuras consistentes con la forma en que las personas hacen y aprenden ciencia.

En esta investigación se pretende vincular el contexto de la localidad donde vive el estudiante para fortalecer la competencia indaga desde la meteorología. La competencia caracterizada como la facultad que tiene una persona de combinar un conjunto de capacidades a fin de lograr un propósito específico en una situación determinada, actuando de manera pertinente y con sentido ético (Ministerio de Educación, 2017), mientras que Reyes y Padilla (2012), resaltan en la competencia la amalgama de conocimientos, habilidades, actitudes y valores que se requieren para resolver y enfrentar diferentes situaciones problemáticas.

El Ministerio de Educación (2016), señala sobre la competencia en ciencias: Indaga mediante métodos científicos para construir sus conocimientos. Mientras que la 
puesta en práctica de esta competencia por parte del estudiante conlleva la realización de las capacidades: Problematiza situaciones para hacer indagación, diseña estrategias para hacer indagación, genera y registra datos o información, analiza datos e información, evalúa y comunica el proceso y resultados de su indagación. A esta competencia la denominamos en el artículo "la competencia indaga."

\section{Metodología}

La investigación se desarrolló con el enfoque cualitativo y en su diseño se empleó la investigación acción (Hernández, Fernández \& Baptista, 2010), pues mediante la investigación se construye el conocimiento, permitiendo que los participantes actúen de manera democrática, donde sus contribuciones son valoradas y se procure la mejora de sus condiciones de vida. En esta investigación participaron voluntariamente estudiantes del segundo grado de educación secundaria, quienes firmaron un consentimiento informado.

Para validar la investigación se recurrió al método de juicio de expertos, fueron tres los especialistas quienes evaluaron y aplicaron una ficha de opinión que contenía nueve criterios con indicadores de valoración. Sobre las capacidades de la Competencia Indaga se especifica:

- Problematizar situaciones para hacer indagación: plantea preguntas sobre hechos y fenómenos naturales, interpretar situaciones y formular hipótesis.

- Diseñar estrategias para hacer indagación: proponer actividades que permitan construir un procedimiento, seleccionar materiales, instrumentos e información para comprobar o refutar las hipótesis.

- Generar y registrar datos o información: obtener, organizar y registrar datos fiables en función de las variables, utilizando instrumentos y diversas técnicas que permitan comprobar o refutar las hipótesis.

- Analizar datos e información: interpretar los datos obtenidos en la indagación, contrastarlos con las hipótesis e información relacionada al problema para elaborar conclusiones que comprueban o refutan las hipótesis.

- Evaluar y comunicar el proceso y resultados de su indagación: identificar y dar a conocer las dificultades técnicas y los conocimientos logrados para cuestionar el 
grado de satisfacción que la respuesta da a la pregunta de indagación (Ministerio de Educación, 2016, p. 284)

Para evaluar el nivel de logro se diseñó una rúbrica para cada una de estas capacidades estableciendo que los niveles logro esperado y logro destacado sean los niveles con el que pretendemos fortalecer la competencia indaga, en la tabla 2 ver las columnas capacidades, logro esperado y logro destacado. En este sentido, la experiencia para la enseñanza de las ciencias, implementación del SME y la ejecución de la estrategia de enseñanza de las ciencias que permite fortalecer la competencia de indagación a través del SME se desarrolló en tres fases:

Fase 3

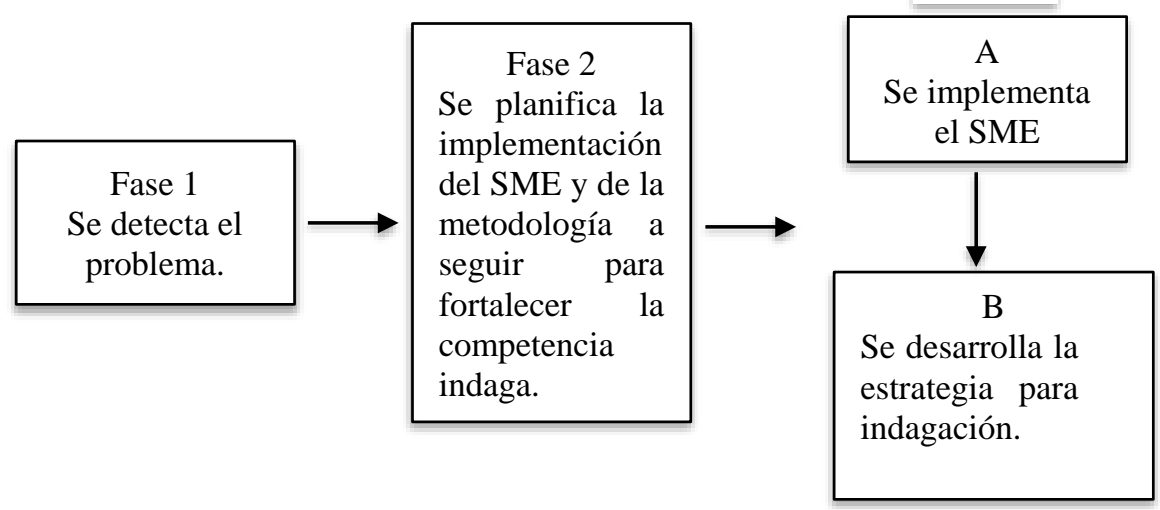

Figura 1. Fases para desarrollar la investigación

\section{Fase 1. Detectar el problema}

El desarrollo de competencias en el área de Ciencia, Tecnología y Ambiente estuvo guiada por el Diseño Curricular Nacional durante los años 2008 al 2014, enfatizaba el desarrollo amplio de contenidos, que se desarrollaba de forma tradicional y no fortalecía la competencia de-indagación, esto constituyó un reto para salir de la rutina, al inicio se trató que los estudiantes se involucren en actividades científicas formando un club de ciencias donde se realizaba experimentos fuera del horario escolar, esto llamó la atención de los estudiantes, ellos se divertían pero no bastaba porque aprender ciencias implica profundizar en la enseñanza mediante la indagación.-En las instituciones educativas rurales de la región Pasco, Perú, el área curricular de Ciencia Tecnología y Ambiente se dispone de materiales 
para experimentación, los mismos que fueron entregados por el Ministerio de Educación o donados por otras instituciones.

En la Institución Educativa San Cristóbal, al realizar el inventario se encontró instrumentos y materiales en el laboratorio que nunca fueron utilizados, perdiéndose oportunidades de aprendizaje para los estudiantes, seguían sellados, estos fueron donados por el gobierno chino en los años 1990 a 1995, entre estos, se ubicó un aparato semejante a un reloj, no contaba con manual de funcionamiento, la simbología empleada indicaba que el instrumento se relacionaba con la atmósfera, posteriormente se identificó que se trataba de un barómetro, instrumento para medir la presión atmosférica. El año 2010 el Gobierno Regional de Pasco, implementó el laboratorio con kits de ciencias, entre ellos se identificó un kit relacionado con el estudio del clima, sin embargo, no disponía del manual correspondiente para su uso adecuado.

\section{Fase 2. Formulación del plan}

\section{Implementación del servicio meteorológico escolar}

En primer lugar, se planificó gestionar recursos económicos para la adquisición, instalación y capacitación de una estación meteorológica convencional ante las entidades públicas que velan por el desarrollo social en este caso la Municipalidad Provincial Daniel Alcides Carrión.

Luego, se sustentó ante el magno Consejo Municipal la importancia de tener una Estación Meteorológica Convencional en la Institución Educativa "San Cristóbal" para realizar trabajos de investigación desde el nivel secundario relacionados con el comportamiento de las condiciones atmosféricas de la zona. Posteriormente, se planificó gestionar el seguimiento, puesta en funcionamiento y respaldo de las autoridades educativas como la Unidad Gestión Educativa Local “Daniel Alcides Carrión.” Finalmente, se planificó gestionar ante la Unidad Gestión Educativa Local "Daniel Alcides Carrión” la adquisición de una estación meteorológica automática.

\section{Implementar la metodología de la indagación}

Se planificó analizar de la modificación parcial del Diseño Curricular Nacional (DCN) que para ese entonces introducía las Rutas de Aprendizaje, que cambió los extensos 
contenidos del DCN por el desarrollo de cuatro competencias entre ellos la competencia indaga. Asimismo, se planificó implementar la estrategia de indagación, teniendo en cuenta el campo temático (nutrición de las plantas, calor y temperatura) de las rutas de aprendizaje que se relacionaba, por estas consideraciones se optó por desarrollarlo con estudiantes del segundo grado.

\section{Fase 3. Implementación del plan}

La investigación se realizó en el contexto de la Institución Educativa "San Cristóbal de Chaupimarca," Tapuc, Pasco, de ámbito rural. Los estudiantes que conformaron el SME comprendieron quince integrantes distribuidos entre el primer y quinto grado. Además, siete estudiantes de segundo grado fueron seleccionados para participar en la estrategia indaga, se incluyeron por invitación y de forma voluntaria.

Primero, el SME se implementó en dos etapas, en la primera etapa, se instaló una caseta meteorológica convencional con instrumentos meteorológicos analógicos. En esta etapa se elaboró un cronograma mensual asignando un turno diario a cada dos estudiantes, la tarea consistió en realizar el recojo de datos meteorológicos cinco veces al día. Se utilizó una planilla meteorológica, se anotaban datos sensoriales (vista) y datos instrumentales (termómetro, pluviómetro, higrómetro entre otros). En la segunda etapa, se implementó el SME, con una Estación Meteorológica Automática, que consta de dos partes, los sensores meteorológicos y el registrador de datos. Segundo, en las etapas de la estrategia de indagación. Se apoyó en el registro de datos del SME, que se desarrolló a través de las siguientes etapas:

\section{Problematización}

Durante el invierno a más de 3500 m.s.n.m. la temperatura ambiente baja drásticamente por las noches, este fenómeno es conocido como la helada y durante el día la humedad relativa disminuye, mientras que la temperatura ambiente aumenta afectando a las plantas y sembríos de los agricultores pues tienden a secarse y marchitarse, afectando la producción. 


\section{Los estudiantes plantean la hipótesis}

Los estudiantes elaboran posibles soluciones a la problemática planteada. Se guía a los estudiantes a establecer relación de causa efecto. Si logramos variar la temperatura ambiental y humedad relativa, esto tendrá un efecto en el crecimiento de las plantas. Se pregunta a los estudiantes ¿qué causa que las plantas se sequen y marchiten en la temporada de invierno? la identificación de la causa se considera la variable independiente mientras que el efecto que tiene sobre las plantas la variable dependiente. Finalmente, los estudiantes plantean la hipótesis: la temperatura ambiental y humedad relativa influye en el crecimiento de las plantas, hojas y tallos.

\section{Diseña estrategias para indagar}

En esta etapa se seleccionan instrumentos adecuados para realizar mediciones. Se pregunta a los estudiantes ¿Qué estrategias podemos utilizar para modificar la temperatura ambiental y humedad relativa? Se decide en consenso como estrategia más pertinente para modificar la temperatura ambiental y humedad relativa el uso de un invernadero, de esta manera influir en el crecimiento de las plantas. Se elige la planta más adecuada para la experimentación.

\section{Genera y registra datos}

Las mediciones de temperatura ambiental, humedad relativa y crecimiento de las hojas y tallos generan datos que es necesario organizarlos a través de tabla. Estos datos se obtienen de mediciones realizadas dentro del invernadero como fuera de ella.

\section{Análisis de datos}

Los estudiantes comparan los datos de la temperatura ambiental, la humedad relativa, crecimiento de las hojas y tallos tanto dentro como fuera del invernadero. Se emplean gráficos de barras.

\section{Evalúa y comunica}

Los estudiantes verifican si la variable independiente influye en la variable dependiente, finalmente exponen sus conclusiones. 


\section{Resultados}

\section{La implementación del Servicio Meteorológico Escolar (SME)}

En octubre de 2014, se implementó una estación meteorológica convencional y se puso en funcionamiento del SME. En primer lugar, fue necesario comprender el funcionamiento de los instrumentos meteorológicos analógicos (termómetro de máxima y mínima, higrómetro, barómetro) que contenía la estación. Nos planteamos el reto de no desperdiciar los materiales sobre meteorología descritos e identificar los medios pertinentes para acondicionar su funcionamiento, y fundamentalmente para desarrollar aprendizajes con los estudiantes.

Al iniciar con la innovación en la enseñanza de las ciencias para el desarrollo de la competencia indaga por medio de la meteorología se tomó en cuenta las Rutas de Aprendizaje (RA), cuyo enfoque está centrado en el desarrollo de competencias. En el año 2016, se logra el tránsito entre lo analógico y lo digital, la Unidad de Gestión Educativa Local "Daniel Alcides Carrión” implementó a la institución con una estación meteorológica automática, esto significó trabajar con parámetros meteorológicos precisos, este equipo está conformado por sensores y un registrador de datos. Siendo la función principal el registro de datos diarios de temperatura ambiental, humedad relativa y otros parámetros meteorológicos.
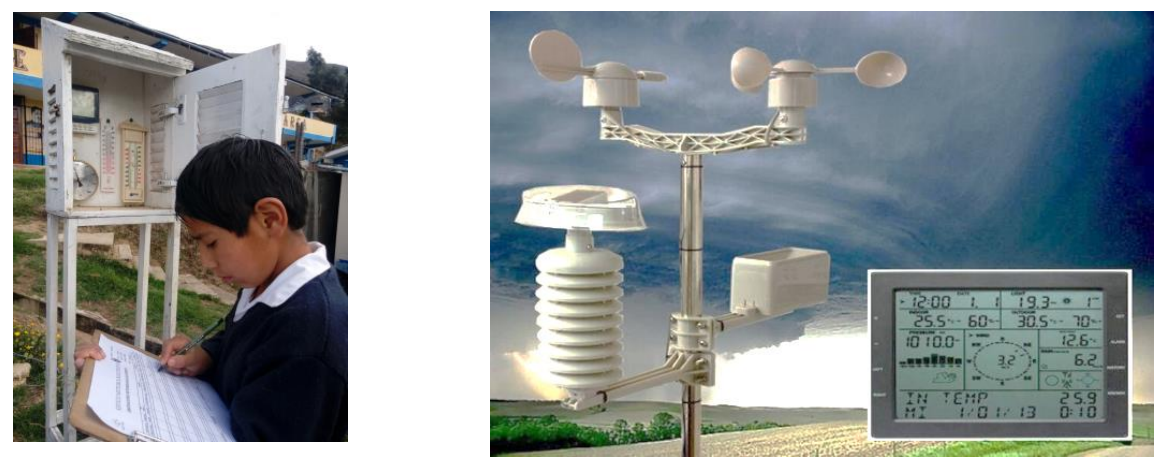

Figura 2. Caseta meteorológica convencional (a la izquierda) y estación meteorológica automática (a la derecha)

Desarrollo de la estrategia de enseñanza de las ciencias para fortalecer la competencia de indagación a través del servicio meteorológico escolar 
La estrategia de la enseñanza en ciencias se realiza en el mes de junio de 2016, el desarrollo del proceso de indagación científica a partir de las actividades realizadas en el SME, toma en cuenta factores como la estación del año en que se realiza la indagación, la ubicación geográfica de la zona y las condiciones atmosféricas.

\section{Problematización}

Al inicio se guía a los estudiantes a describir los sembríos de sus padres y agricultores, preguntando ¿por qué se secan y marchitan entre los meses de junio-agosto? Observan las características de las plantas de la intemperie, en el patio del colegio y de sus sembríos, en esta parte se pretende que el estudiante argumente que: (a) los cambios drásticos de temperatura ambiental, humedad durante el día y la noche en época de invierno. Durante el día el cielo está completamente despejado y por las noches se presentan las heladas, en cuanto a la humedad en horas del día es baja debido a que el cielo se encuentra despejado. En ese sentido pueden emplear expresiones como la temperatura sube, baja, hace mucho frío, mucho calor, mucha humedad. (b) el estado de las plantas que se encuentran en la intemperie, en esta temporada, la mayoría de plantas se secan y se marchitan. Algunas anotaciones de los estudiantes son:

Las plantas que se encuentra en la intemperie presentan un color amarillo debido a que están marchitas,

Las plantas que están cerca de las paredes conservan el color verde.

Para confirmar las observaciones se recurre a los datos que se registran diariamente en el SME, analizando la variación de la temperatura ambiental y humedad relativa.

\section{Los estudiantes plantean la hipótesis}

Identificada la problemática las plantas se marchitan y secan entre los meses de junioagosto. Se pregunta ¿Qué causa que las plantas se sequen y marchiten en estos meses? Se pide a los estudiantes elaborar posibles respuestas, siempre teniendo en cuenta la relación causa efecto entre la temperatura ambiental, humedad relativa y el crecimiento de las plantas. Nuevamente se pregunta: ¿La temperatura ambiental y humedad relativa influye en el crecimiento de las plantas? o ¿El crecimiento de las plantas influye en la temperatura ambiental y humedad relativa? 
Los estudiantes concuerdan que: La temperatura ambiental y la humedad relativa influyen en el crecimiento de las plantas. Se explica que la temperatura ambiental y la humedad relativa se considera la variable independiente, mientras que el crecimiento de las plantas se considera la variable dependiente. El crecimiento de las plantas se considera variable dependiente porque el crecimiento de las plantas depende de la temperatura ambiental y la humedad relativa. Finalmente se explica a los estudiantes que lo planteado la temperatura ambiental y la humedad relativa influyen en el crecimiento de las plantas, se considera la hipótesis de trabajo

\section{Diseña estrategias para indagar}

Teniendo en cuenta la hipótesis, se plantea a los estudiantes la pregunta ¿Cómo se logrará demostrar lo afirmado en la hipótesis?, en nuestro caso ¿cómo demostraremos que la temperatura ambiental y la humedad relativa afectan al crecimiento del rabanito? Luego de haber planteado que la temperatura ambiental y la humedad relativa afectan al crecimiento de las plantas se pide que respondan a la pregunta ¿Qué estrategia podemos utilizar para modificar la temperatura ambiental y humedad relativa? Los estudiantes refieren que durante la temporada de heladas sus padres utilizan paja para cubrir sus hortalizas y así protegerlos del sol intenso para que no les seque y de las heladas para que no las congele; esto representa los saberes propios que el poblador agrícola posee en el manejo de sus cultivos, entonces, se aprovecha el momento del diálogo para introducir la idea del invernadero.

De acuerdo a la experiencia en sus huertos familiares los estudiantes acuerdan utilizar protección para conservar la temperatura ambiental y humedad relativa por lo que deciden recurrir al invernadero pequeño armable que posee la institución educativa. La institución educativa también posee un huerto que es utilizado anualmente para producir hortalizas el cual está cubierto con malla raschel para proteger las plantas de las heladas o granizos que pudieran presentarse durante el año.

Los estudiantes describen las características que presentan las plantas que se encuentran dentro del huerto y las que están en la intemperie; una de las razones que exponen los estudiantes es que dentro del huerto como hay sombra entonces la temperatura ambiental es menor por eso las plantas están más verdes, y para comprobar esta afirmación se les pide medir la temperatura con un termómetro ambiental en ambos lados, ellos encuentran una ligera diferencia de dos grados Celsius, en este caso mayor dentro del huerto, lo que descarta 
su afirmación, por lo que se realiza el mismo procedimiento con el higrómetro y barómetro, encontrando una ligera diferencia en el primero y ninguna con el segundo instrumento, en tal sentido optan por usar el termómetro ambiental y el higrómetro para medir la temperatura ambiental y la humedad relativa, como instrumentos para medir la variable independiente (temperatura ambiental y humedad)

Se solicita a los estudiantes que propongan una planta para la experimentación, se prioriza el tiempo que tarda en crecer, finalmente se acuerda utilizar el rabanito por ser una planta de crecimiento rápido ya que le toma 60 días desde la siembra hasta obtener el producto. Para medir el crecimiento de las hojas y tallo del rabanito se decide utilizar una regla.

A continuación, se procede a implementar el área de experimentación: a) Armar el invernadero, b) preparar el terreno dentro y fuera del invernadero, c) sembrar los rabanitos en ambos terrenos, d) colocar dentro del invernadero el termómetro ambiental, el higrómetro y la regla, e) realizar las mediciones temperatura ambiental, humedad relativa y crecimiento de las hojas y tallos del rabanito en ambos terrenos todos los días, a las 8:00 horas.

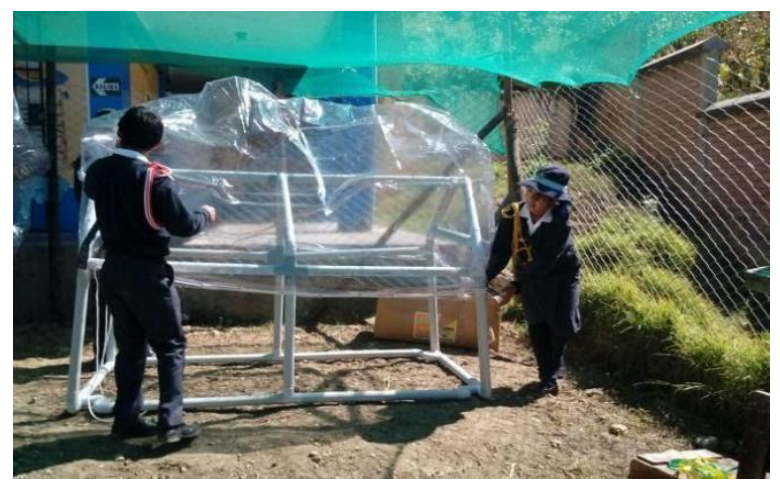

Figura 3. Armado el invernadero en el área de experimentación

\section{Genera y registra datos}

Se pide a los estudiantes elaborar una tabla con las mediciones señaladas en e), considerando las unidades correspondientes. Los estudiantes registran las mediciones durante el mes de junio de 2016 en un lapso de 30 días. Se dispone del registro completo de todos los días. A modo de ejemplo se muestran los datos de los tres primeros y últimos días. 
Tabla 1

Tabla de registro de datos

\begin{tabular}{|c|c|c|c|c|c|c|c|c|c|}
\hline \multirow[t]{3}{*}{ Fecha } & \multirow[t]{3}{*}{ Hora } & \multicolumn{4}{|c|}{ Fuera del invernadero } & \multicolumn{4}{|c|}{ Dentro del invernadero } \\
\hline & & \multirow{2}{*}{$\begin{array}{c}\text { Temperatura } \\
\text { Ambiental } \\
\left({ }^{\circ} \mathbf{C}\right)\end{array}$} & \multirow{2}{*}{$\begin{array}{c}\text { Humedad } \\
\text { Relativa } \\
(\%)\end{array}$} & \multicolumn{2}{|c|}{$\begin{array}{l}\text { Crecimiento del } \\
\text { rabanito }\end{array}$} & \multirow{2}{*}{$\begin{array}{c}\text { Temperatura } \\
\text { ambiental } \\
\left({ }^{\circ} \mathrm{C}\right)\end{array}$} & \multirow{2}{*}{$\begin{array}{c}\text { Humedad } \\
\text { Relativa } \\
(\%)\end{array}$} & \multicolumn{2}{|c|}{$\begin{array}{l}\text { Crecimiento del } \\
\text { rabanito }\end{array}$} \\
\hline & & & & $\begin{array}{l}\text { Hoja } \\
(\mathbf{m m})\end{array}$ & $\begin{array}{l}\text { Tallo } \\
(\mathbf{m m})\end{array}$ & & & $\begin{array}{l}\text { Hoja } \\
(\mathbf{m m})\end{array}$ & $\begin{array}{l}\text { Tallo } \\
\text { (mm) }\end{array}$ \\
\hline $2016-06-1$ & 08:00 & 10,3 & 58 & 2 & 5 & 12 & 60 & 4 & 1 \\
\hline $2016-06-2$ & 08:00 & 9,1 & 99 & 3 & 5 & 9 & 54 & 4 & 1 \\
\hline $2016-06-3$ & 08:00 & 8 & 99 & 5 & 7 & 9 & 46 & 5 & 2 \\
\hline $2016-06-28$ & 08:00 & 9,3 & 60 & 27 & 22 & 11 & 60 & 30 & 30 \\
\hline 2016-06-29 & 08:00 & 10.8 & 52 & 30 & 25 & 12 & 57 & 35 & 33 \\
\hline 2016-06-30 & 08:00 & 11,3 & 46 & 30 & 25 & 12 & 49 & 38 & 35 \\
\hline
\end{tabular}

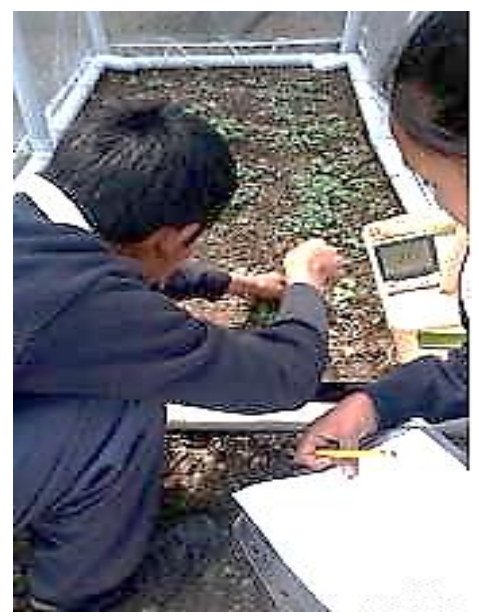

Figura 4. Registro de datos

\section{Análisis de datos}

Se guía a los estudiantes a elaborar (a) gráficos de barras (ver figura 5); y (b) comparar de la temperatura ambiental, humedad relativa y crecimiento de las hojas y tallos del rabanito, dentro y fuera del invernadero.

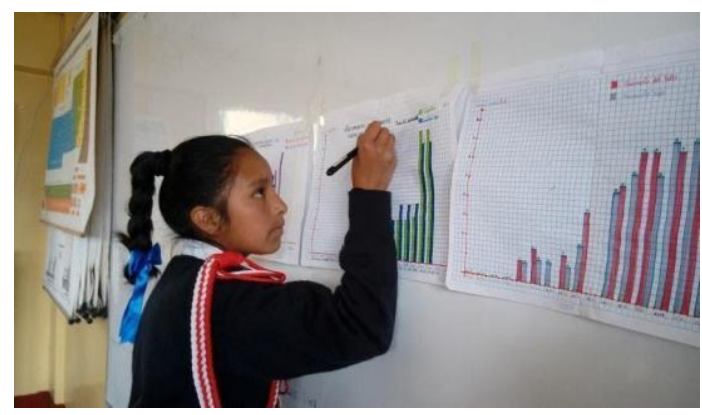

Figura 5. Elaborando gráfico de barras 


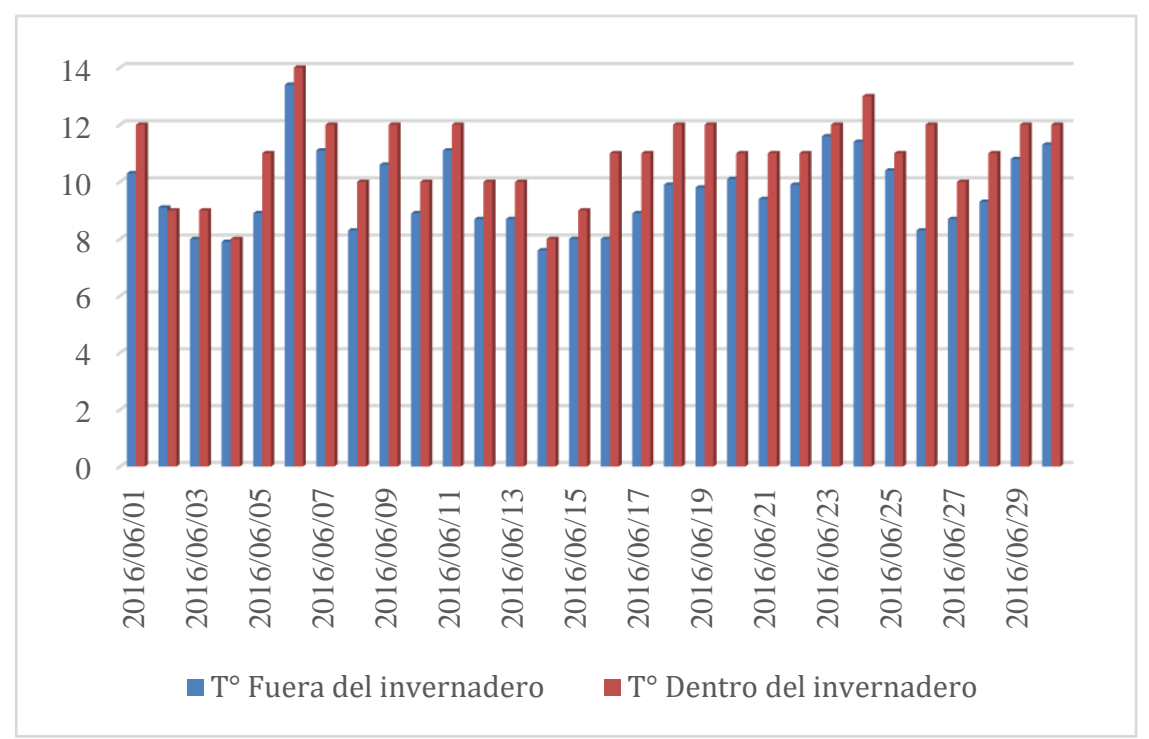

Figura 6. Temperatura Ambiental

Interpretación: Se observa que dentro del invernadero la temperatura es siempre mayor que fuera de ella.

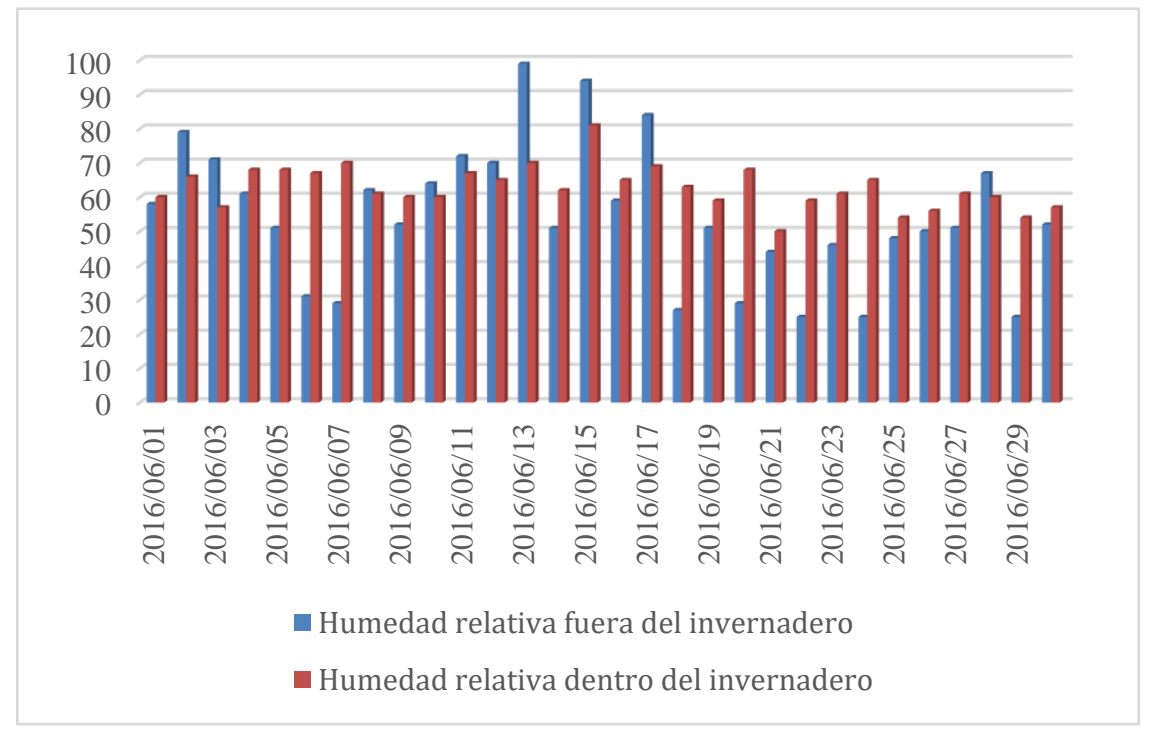

Figura 7. Humedad relativa

Interpretación: Se observa que dentro del invernadero la humedad relativa se mantiene en un promedio de $62,9 \%$, mientras que fuera del invernadero la humedad disminuye y aumenta de manera significativa. 


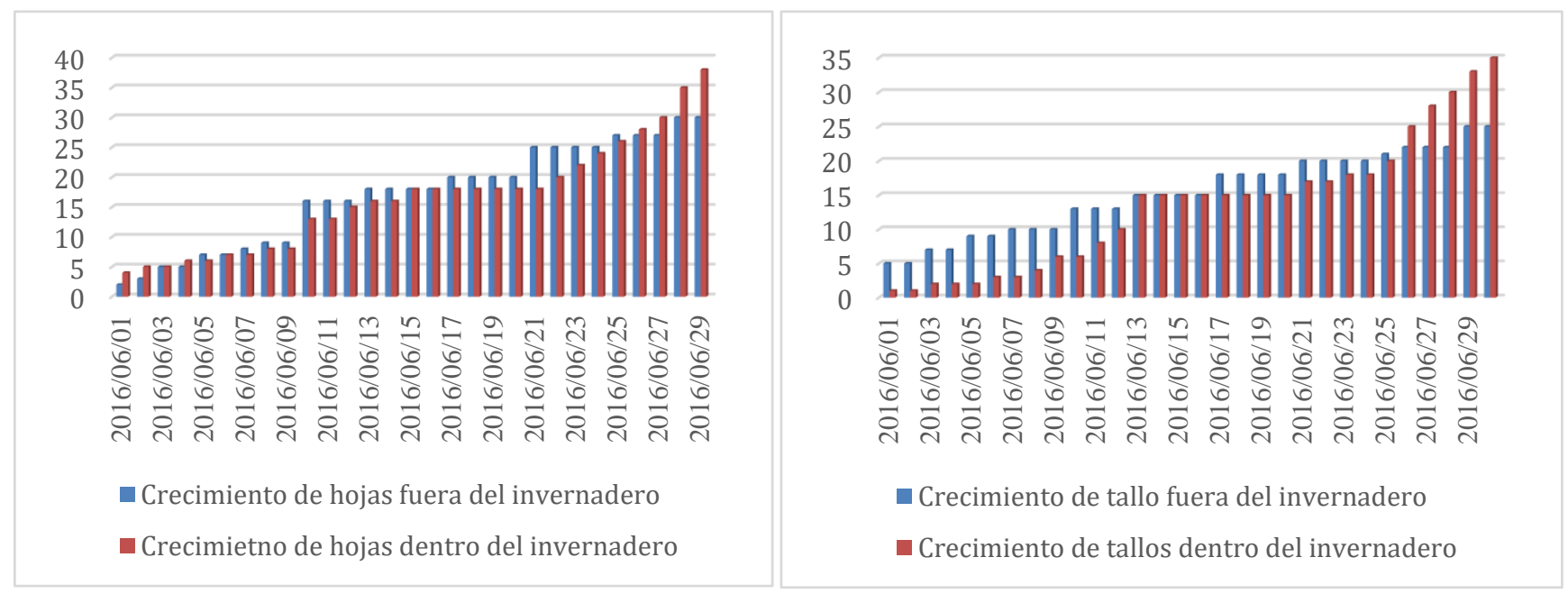

Figura 8. Crecimiento de hojas y tallo

Interpretación: Al iniciar la toma de datos el crecimiento de las hojas del rabanito fuera del invernadero es mayor, sin embargo, al transcurrir el tiempo el crecimiento de las hojas dentro del invernadero es mayor. El crecimiento de los tallos del rabanito fuera del invernadero, al iniciar la toma de datos superan a los que se encuentran dentro del invernadero, pero a partir del día quince logra igualar su crecimiento y cuando llega al día veintinueve los tallos de los rabanitos dentro del invernadero logran superar en crecimiento.

\section{Evalúa y comunica}

Los estudiantes, en base a los datos obtenidos sobre el crecimiento de las hojas y tallos del rabanito, la temperatura ambiental y humedad relativa, dentro y fuera del invernadero establecen lo siguiente: La temperatura ambiental influye en el crecimiento de las plantas; La humedad relativa influye en el crecimiento de las plantas. A continuación, los estudiantes confirman la hipótesis. Durante el mes de junio la temperatura ambiental y la humedad relativa influyen en el crecimiento de las plantas. Finalmente, los estudiantes exponen sus resultados a sus compañeros.

\section{Discusión}

Mediante la estrategia de enseñanza de las ciencias y del SME fue posible promover en los estudiantes el tránsito por las etapas de la indagación a) Problematización; b) Diseña 
estrategias para indagar; c) Genera y registra datos; d) Analiza datos e) Evalúa y comunica. Los niveles de logro que alcanzaron los participantes en cada una de las capacidades de la Competencia Indaga se detallan en las columnas "evidencias" de la tabla 2.

Tabla 2

Rúbrica para evaluar los niveles alcanzados en las capacidades de la Competencia Indaga 


\section{NIVELES DE LOGRO DE LOS PARTICIPANTES QUE FORTALECEN LA COMPETENCIA INDAGA}

CAPACIDADES

\section{LOGRO ESPERADO}

Evidencias

Con ayuda, formula preguntas acerca de las variables que influyen en el crecimiento de la planta, y sin dificultad selecciona aquella que puede ser indagada científicamente.

Con ayuda plantea hipótesis en las que sin dificultad establece relaciones causalidad entre las variables.

Con ayuda y sin dificultad propone procedimientos para observar, manipular la variable

independiente, medir la variable dependiente y controlar aspectos que modifican la experimentación.

Diseña estrategias para hacer indagación

Con ayuda y sin dificultad selecciona herramientas,

materiales

instrumentos

recoger para cualitativos/ cuantitativos. Prevé el tiempo y las medidas de seguridad personal y del lugar de trabajo.

Genera y registra datos $\mathrm{e}$ información

Analiza datos e información

Con ayuda y sin dificultad obtiene datos cuantitativos a partir de la manipulación de la variable independiente y mediciones repetidas de la variable dependiente. Controla aspectos que modifican experimentación.
Joseph propone una pregunta diciendo: ¿Por qué el rabanito no crece bien?, (se le indica que, debe considerar en su pregunta qué es aquello que le impide a la planta crecer), Rusbel menciona su pregunta ¿Por qué cuando cae hielo se secan las plantas?

Mirtha: las plantas que están cerca de las paredes no se marchitan siguen verdes, esto porque le hacen sombra y la helada no les llega.

Con indicaciones estudiantes consideran:

$\operatorname{los}$

-Sembrar la planta dentro y fuera del invernadero

-Medir la temperatura ambiental y la humedad relativa.

-Medir el crecimiento del rabanito.

Los estudiantes listan Semilla abono Pico rastrillo invernadero Regla termómetro higrómetro

Con ayuda compara Traza el plano cartesiano, los datos obtenidos ubica en eje X el tiempo y en el cuantitativos para eje $Y$ las variables establecer relaciones de causalidad; y sin dificultad contrasta los resultados con su

Anota la medida de la temperatura ambiente, humedad relativa $y$ crecimiento de hojas y tallos del rabanito a las horas establecidas. (figura 6, 7 y 8) (crecimiento de hojas y tallos del rabanito, temperatura ambiente y humedad relativa), se les indica que comparen
De manera autónoma, formula preguntas acerca de las variables que influyen en el crecimiento de la planta, y sin dificultad selecciona aquella que puede ser indagada científicamente.

De manera autónoma plantea hipótesis en las que sin dificultad establece relaciones de causalidad entre las variables.

De manera autónoma y sin dificultad selecciona herramientas, materiales e instrumentos para recoger datos cualitativos/ cuantitativos. Prevé el tiempo y las medidas de seguridad personal y del lugar de trabajo.

De manera autónoma y sin dificultad obtiene datos cuantitativos a partir de la manipulación de la variable independiente y mediciones repetidas de la variable dependiente. Controla aspectos que modifican la experimentación.

De manera autónoma compara los datos obtenidos cuantitativos para establecer relaciones de causalidad; y sin dificultad contrasta los resultados con su hipótesis e información

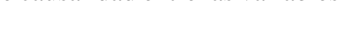

Mencionan que el problema radica en que las bajas temperaturas y la humedad relativa afectan a los sembríos y todas las demás plantas.

Afirman en grupo que lo que influye en el crecimiento de las plantas durante el mes de junio son las bajas temperaturas del ambiente y la variación de la humedad relativa, y diferencian las variables.

Para la parte experimental consideran:

Armar el invernadero

Preparar la tierra.

Sembrar la planta dentro y fuera del invernadero.

Regar las plantas

Medir la temperatura ambiental y la humedad relativa

Medir el crecimiento del rabanito

Consideran en la lista de materiales

Semilla, abono.

Pico rastrillo, regadera, invernadero.

Regla, termómetro, higrómetro.

Se organizan por turnos para anotar la medida de la temperatura ambiente, humedad relativa y crecimiento de hojas y tallos del rabanito a las horas establecidas.
Traza el plano cartesiano, ubica en eje X el tiempo y en el eje Y las variables (crecimiento de hojas y tallos del rabanito, temperatura ambiente $\mathrm{y}$ humedad relativa), comparan entre los datos de dentro y fuera 


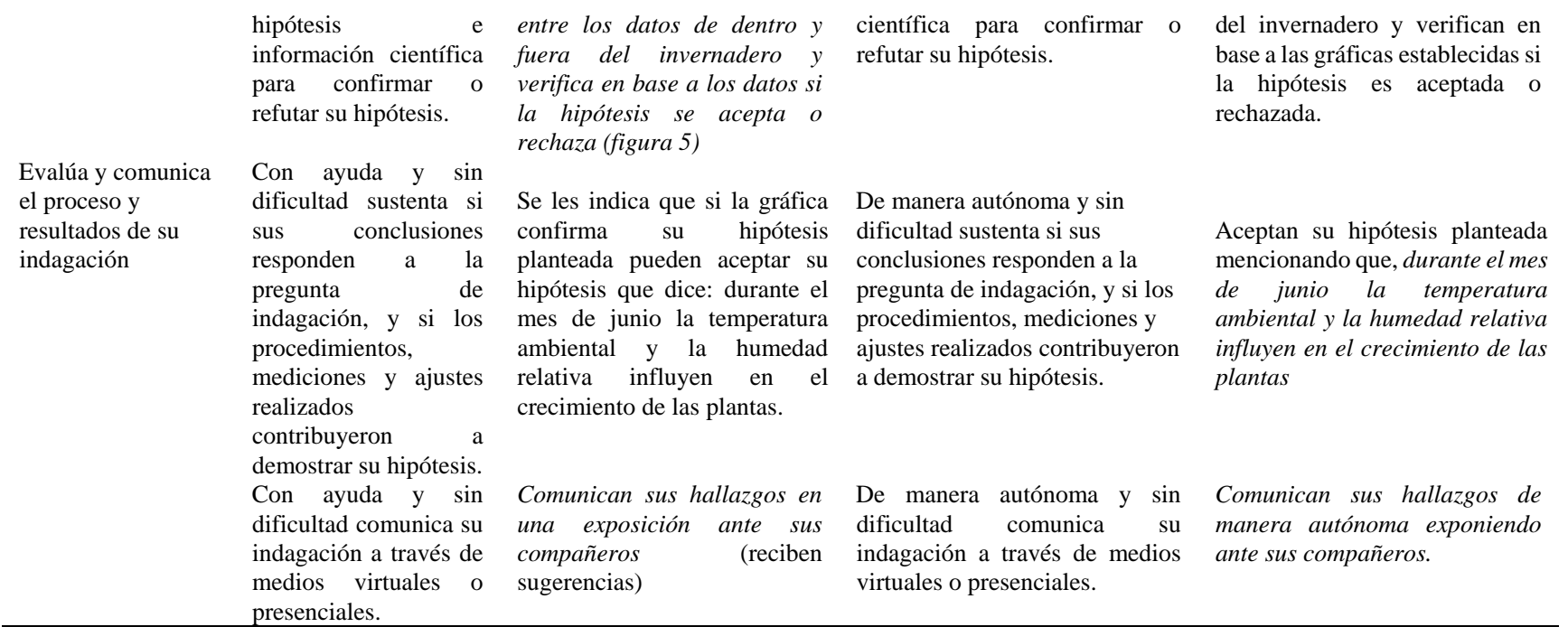

Para conseguirlo fue fundamental proporcionar espacios a los estudiantes distintos a los que se les brinda en un proceso de aprendizaje tradicional. En esta línea hay investigaciones que realizan esfuerzos similares procurando fortalecer la competencia indaga (Barrera \& Crisancho, 2017), se propugna “[...] incidir en la enseñanza de ciencias en las escuelas básica y media, su objetivo es fomentar en los niños competencias científicas y tecnológicas, habilidades de comunicación y competencias ciudadanas" (Meisel et al., 2010, p. 114). En este aspecto, es vital la guía del docente en relación al objetivo de la investigación, en ese sentido se coincide con la consideración de Sala y Font (2019) que es papel del maestro que permite gestionar y tomar decisiones alineados con los propósitos de la investigación.

En esta investigación tanto la innovación de enseñanza de las ciencias con el SME como la estrategia basada en ECBI fue desarrollada con escolares de nivel medio. Es en la etapa escolar que el estudiante se formula preguntas de los problemas que aquejan su entorno y su vida cotidiana, fue fundamental encauzar su curiosidad a través de la estrategia de enseñanza de las ciencias, López (2014) refiere a través de la indagación es posible potenciar la curiosidad, la indagación y motivar las preguntas, eso es diferente de aprender memorizando respuestas.

Aprovechamos la curiosidad inherente al estudiante, sobre lo cual existe un consenso en su inicio desde la niñez (National Academy of Sciences, 2000; Dyasi, 2014), curiosidad que expresa a través de preguntas del ¿cómo? ¿porqué? cuestionando los fenómenos que se les presenta. Este deseo intenso por averiguar, por saber, es un atributo humano fundamental 
(Dyasi, 2014). Al respecto, diversos estudios enfocan su interés en desarrollar la estrategia ECBI en escolares de nivel básico y medio enfatizan el papel del estudiante como sujeto activo, autónomo y responsable de su aprendizaje, desarrollando también actitudes y valores (Sala y Font, 2019; Martínez \& Olcina, 2019; Castillo, 2016; Crujeiras \& Cambeiro, 2018; Pozuela \& Cascarroza, 2018; Ibáñez, Aguilar \& Solé, 2019, Reyes-Cárdenas \& Padilla, 2012).

Los participantes del estudio transitan mediante la guía del maestro por las etapas de la estrategia ECBI: problematización, plantear hipótesis, diseñar estrategias para indagar, generar y registrar datos, análisis de datos, evaluar y comunicar, por ello, demostramos que su ejecución es factible a través de la meteorología contribuyendo a fortalecer la competencia indaga en tanto el programa escolar plantea: Indagar científicamente es conocer, comprender y usar los procedimientos de la ciencia para construir o reconstruir conocimientos. De esta manera, los estudiantes aprenden a plantear preguntas o problemas sobre los fenómenos, la estructura o la dinámica del mundo físico. Movilizan sus ideas para proponer hipótesis y acciones que les permitan obtener, registrar y analizar información que luego comparan con sus explicaciones. Involucra, también, una reflexión sobre los procesos que se llevan a cabo durante la indagación, a fin de entender la ciencia como proceso y producto humano que se construye en colectivo. (MINEDU, 2016)

Algunos estudios reportan de acuerdo a sus propósitos y de manera contextualizada el tránsito por todas las etapas de la estrategia ECBI (Castillo, 2016; Sbarbati, 2015; Torres, Caldeiro \& Maeots, 2020). Del mismo modo, en esta investigación fue fundamental la generación y registro de datos proporcionados por el SME como soporte para la estrategia de enseñanza de las ciencias que fortalece la competencia indaga. En esta misma línea, se reporta que la enseñanza de las ciencias a través de la meteorología brinda la posibilidad de recogida de datos in situ, es adecuada (Martínez \& Olcina, 2019), además potencia la observación al favorecer la realización de descripciones precisas (Romgnoli \& Sebben, 2015)

\section{Conclusiones}

La experiencia de innovación en la enseñanza de las ciencias ha sido descrita en primer lugar tomando como ámbito de la investigación una institución educativa rural de la región Pasco, Perú. Previo a fortalecer la competencia de indagación se implementó el SME a la que hemos denominado iniciativa de innovación de enseñanza de las ciencias ya que se 
toma la decisión de resolver la problemática en dicho contexto, de una enseñanza en ciencias basada en contenidos a otra que fortalezca la competencia indaga. Por esta razón, se gestiona ante las autoridades locales la puesta en funcionamiento de la estación meteorológica analógica y automática. Alrededor del empleo de las estaciones se organiza el SME, involucrando a escolares en el registro de datos meteorológicos.

La estrategia de la enseñanza de las ciencias permite fortalecer la competencia indaga en los estudiantes participantes del estudio porque a través de ella fueron capaces de comprender el proceso de la indagación desde la problematización, diseñando estrategias para indagar, recopilando y analizando datos para finalmente arribar a conclusiones y comunicar los resultados, por lo tanto, se ha podido demostrar en esta investigación que la estrategia de enseñanza en ciencias es factible de realizarse por medio de la meteorología.

\section{Referencias}

Barrera, Y., \& Cristancho, R. (2017). Desarrollo de la competencia indagación en Ciencias Naturales. Educación y Ciencia, 20, 27-41. http://repositorio.uptc.edu.co/handle/001/2468

Castillo, C. E. (2016). El cuento como estrategia para desarrollar la indagación en ciencias naturales. Educación y Ciencia, 20, 61-76. https://revistas.uptc.edu.co/index.php/educacion_y_ciencia/article/view/8898 Doi:10.5354/2452-5014.2021.60683

Crujeiras, B., \& Cambeiro, F., (2018) Una experiencia de indagación cooperativa para aprender ciencias en educación secundaria. Revista Eureka sobre Enseñanza y Divulgación de las Ciencias, 15 (1), 1201. https://revistas.uca.es/index.php/eureka/article/view/3490

Doi: 10.25267/Rev_Eureka_ensen_divulg_cienc.2018.v15.i1.1201

Dyasi, H. (2014). La enseñanza de la Ciencia en la Educación Básica. Colonia del Valle: INNOVEC. https://innovec.org.mx/home/images/antologia\%20sobre\%20indagacionvol.1.pdf

Educación en Ciencia Basada en la Indagación. (2015). Método indagatorio. ECBI Chile. http://www.ecbichile.cl/home/metodo-indagatorio/ 
Harlen, W. (2013). Evaluación y educación en ciencias basada en la indagación: Aspectos de la política y la práctica. Trieste: Global Network of Science Academies (IAP) y Science Education Programme (SEP).

https://www.interacademies.org/sites/default/files/publication/assessment_guide spanish_0.pdf

Hernández, R., Fernández, C., \& Baptista, P. (2010). Metodología de la investigación. México D.F.: Mc Graw Hill.

López, P. (2014). El cuaderno de ciencias en la clase indagatoria. En Gutierrez, R., Everaert, C. \& Robles C. (Eds), Antología sobre la indagación (pp. 51-57). México D.F.: Innovación y Enseñanza de la Ciencia A. C.

http://www.innovec.org.mx/home/images/antologia\%20sobre\%20indagacion-

vol.1.pdf

Martínez, L., \& Olcina, J., (2019). La enseñanza escolar del tiempo atmosférico y del clima en España currículo educativo y propuestas didácticas. Anales de Geografía de la Universidad Complutense, $39 \quad$ (1), 125-148. http://dx.doi.org/10.5209/AGUC.64680

Meisel, J., Helga, B., Saavedra, C., \& Patiño, L. (2010). El éxito en la enseñanza de las ciencias basada en indagación (ECBI): Una cuestión más allá del aula de clase. Pedagogía y Saberes, 32, 11-124. https://doi.org/10.17227/01212494.32pys111.124

Ministerio de Educación (2017). Currículo Nacional de la Educación Básica Regular. Lima: Ministerio de Educación.

Ministerio de Educación (2016). Programa Curricular de Educación Secundaria. Lima: Ministerio de Educación.

Molina-Ruiz, N., \& Gonzales-García, P. (2021). Ciencias naturales y aprendizaje socioemocional: una experiencia desde la enseñanza de las ciencias basada en la indagación. Revista Saberes Educativos, 6, 25-58. Doi: 10.5354/24525014.2021.60683

National Academy of Sciences (2000). Inquiry and the National Science Education Standards: A Guide for Teaching and Learning. Washington, D.C.: National Academy Press.

Pozuela, J., \& Cascarroza, E. (2018) Inmersión en el mundo de la nano-ciencia a través de una experiencia de indagación guiada con alumnos de Educación Secundaria. Rei Do Crea, 7, 376-387. https://dialnet.unirioja.es/servlet/articulo?codigo=7179920

Reyes-Cárdenas, F., \& Padilla, K. (2012). La indagación y la enseñanza de las ciencias. Educ. quím, 23 (4), 415-421. https://doi.org/10.1016/S0187-893X(17)30129-5 
Romagnoli, C., \& Sebben, V., (2015). Nociones de Física de la Atmósfera en la Escuela Primaria. Una mirada desde la Meteorología Escolar. Revista de enseñanza de la física. 27, 741-747. www.revistas.unc.edu.ar/index.php/revistaEF/

Sbarbati, N., (2015). Educación en ciencias basada en la indagación. Revista Iberoamericana de Ciencia, Tecnología y Sociedad, $10, \quad$ 1-10. https://www.redalyc.org/pdf/924/92433772001.pdf

Sala, G., \& Font, V. (2019). El papel de la modelización en una experiencia de enseñanza de matemáticas basadas en indagación. Avances de Investigación en Educación Matemática, 16, 73-85. https://doi.org/10.35763/aiem.v0i16.283

Ibañes, M., Aguilar, D., \& Solé A, (2019). Las ayudas en indagaciones científicas escolares mediadas por herramientas tecnológicas Investigaciones de la última década. Digital Education Review, 36, 223-242. https://doi.org/10.1344/der.2019.36.223-242

Torres, A., Caldeiro, M. \& Maeots, M. (2020). Aprendizaje basado en el contexto español. Luz. XIX. (3), 3-18. https://luz.uho.edu.cu/index.php/luz/article/view/1056

Uzcátegui, Y., \& Betancourt, C. (2013). La metodología indagatoria en la enseñanza de las ciencias: una revisión de su creciente implementación a nivel de Educación Básica y Media. Revista de Investigación, $37 \quad$ (78), 109-127. http://ve.scielo.org/scielo.php?script=sci_arttext\&pid=S101029142013000100006\& $\underline{\operatorname{lng}=\text { es \& tlng=es. }}$ 